\title{
The relevance of assessment of intestinal P-gp inhibition using digoxin as an in vivo probe substrate
}

\author{
Jack G. Shi, Yan Zhang and Swamy Yeleswaram
}

The recent Review (Membrane transporters in drug development. Nature Rev. Drug Discov. 9, 215-236; 2010) ${ }^{1}$ by the International Transporter Consortium (ITC) proposed that, for a new molecular entity (NME) intended for oral administration, a clinical drug interaction study with digoxin should be conducted if [I2]/IC $\mathrm{I}_{50}$ $\geq 10$ (where $\mathrm{I} 2$ is the hypothetical concentration of the NME in gut lumen, and $\mathrm{IC}_{50}$ is the NME's potency of P-glycoprotein (P-gp) inhibition in vitro). However, the Review does not adequately address the choice of digoxin as a suitable in vivo P-gp probe substrate in the context of digoxin's clinical pharmacokinetics, as well as the prevalence of drug-drug interactions due to inhibition of P-gp at the intestinal level and hence the importance of such interactions.

Digoxin is certainly a sensitive probe substrate of P-gp in in vitro studies, as evidenced by a high efflux ratio across Caco-2, MDR1-MDCK and MDR1-LLCPK1 cells, as well as effective inhibition of efflux in the presence of P-gp inhibitors. However, the in vivo situation is less clear. In humans, digoxin is subjected to minimal metabolism and its oral bioavailability approximates its fraction absorbed in the gastrointestinal tract. Administered in standard tablet formulation, digoxin exhibits good but incomplete absorption; for example, the absolute bioavailability of Lanoxin (GlaxoSmithKline) is 60-80\% (REF. 2). When dosed in solubilized formulations (for example, Lanoxicaps), digoxin is almost completely absorbed ${ }^{2,3}$, which questions the relevance of P-gp-mediated efflux in the oral absorption of digoxin. Other reports support the notion that the rate of oral absorption of digoxin is limited by dissolution (rather than permeability $)^{4,5}$. It should also be noted that grapefruit juice, an intestinal P-gp inhibitor, has a non-significant effect on digoxin serum concentrations ${ }^{6}$, and ketoconazole - a potent P-gp inhibitor - is not known to cause significant interaction with digoxin, which further undermines the choice of digoxin as an in vivo probe substrate.
Numerous reported clinical drug interactions involving digoxin and P-gp inhibitors (verapamil, clarithromycin, intraconazole, carvedilol, talinolol, nifedipine and propafenone) that are used as evidence of intestinal P-gp inhibition suffer from a critical flaw. The formulation of each of these interacting drugs contains polyethylene glycol (PEG) and/or propylene glycol (PG) as excipients. It is therefore reasonable to suspect that these interactions may have little to do with P-gp inhibition and more to do with solubilization of digoxin. It is worth noting that digoxin oral absorption is not affected when dosed in solubilized formulations, in which PEG or PG is used as a typical co-solvent ${ }^{2,3,7}$.

Consistent with dissolution rate-limited oral absorption of digoxin, which has been well established, there is no compelling example that we could find in the literature to clearly substantiate clinical drug interaction for digoxin due to inhibition of intestinal P-gp ${ }^{8}$. Therefore, a digoxinbased clinical study with the objective of evaluating drug interaction effect resulting from intestinal P-gp inhibition is unlikely to produce any meaningful data. On the other hand, there is credible evidence to suggest that solubilizing or emulsifying excipients such as PEG, PG and cremophor ${ }^{9}$ may significantly increase digoxin oral absorption and serum concentrations. Due to the narrow therapeutic index of this widely prescribed drug, we think a clinical interaction study with digoxin should be mandatory for any investigational drug product with an oral formulation containing solubility-enhancing excipients such as those discussed above, regardless of the P-gp-inhibitory potential of the active principal ingredient itself.

We recognize the need in drug development to evaluate P-gp inhibition at the gut level as a potential source of drug interaction for NMEs that are potent P-gp inhibitors in vitro, and suggest the following set of criteria for selecting the ideal P-gp probe for clinical evaluation of drug-drug interactions. First, an ideal probe substrate for P-gp should be a sensitive substrate of $\mathrm{P}$-gp, as evidenced by its high efflux ratios in bidirectional transport assays in P-gpexpressing cell lines; second, it should be dosed low enough such that the initial concentration in the gut does not exceed its $K_{\mathrm{m}}$ for P-gp, thereby enabling P-gp-mediated efflux in vivo; third, the P-gp probe substrate should exhibit permeability (rather than solubility or dissolution) rate-limited oral absorption and a low-to-moderate oral bioavailability; fourth, it should be minimally metabolized (including firstpass metabolism at the gut wall), so that the pharmacokinetics is not confounded by processes affecting metabolism; fifth, it should not be a high-affinity substrate for any uptake or efflux transporter (other than P-gp) that is expressed in human intestinal enterocytes; and last, it should have adequate clinical experience from a safety standpoint. Digoxin does not meet the third requirement, and thus is not an acceptable probe drug for the purpose.

A survey of the literature indicates that molecules that possess all these qualities are rare. The $\beta$-blocker drug talinolol is a wellstudied P-gp substrate with approximately tenfold efflux ratio in Caco- 2 cells ${ }^{10}$, a moderate oral bioavailability of approximately $55 \%$ for the immediate-release (IR) dosage, and negligible $(<1 \%)$ metabolic clearance ${ }^{11}$. The limited oral bioavailability of talinolol IR tablets is unlikely to be caused by slow dissolution, as the tablet exhibits relatively rapid dissolution in aqueous media with no less than $80 \%$ of the labelled amount of the drug substance dissolved in 30 minutes at pH 6.8 and almost $100 \%$ of the drug dissolved in 10 minutes at pH 1.0 (REF. 12). With concomitant administration of erythromycin, a P-gp inhibitor, talinolol exhibited increased oral bioavailability without any change in renal clearance ${ }^{13}$ whereas, following intestinal P-gp induction by rifampin, the oral bioavailability of talinolol was significantly decreased ${ }^{14}$, suggesting that intestinal P-gp-mediated efflux is the rate-limiting factor for oral absorption of talinolol. Although talinolol is also a substrate of certain uptake transporters expressed in human intestines ${ }^{15}$, its overall profile seems to be acceptable as an in vivo probe substrate of intestinal P-gp. Besides talinolol, there may be proprietary molecules that meet the above criteria, and we urge the ITC to encourage pharmaceutical companies to voluntarily contribute and share the data for potential model P-gp substrates in the quest to find an ideal probe P-gp substrate. 


\section{CORRESPONDENCE}

Jack G. Shi, Yan Zhang and Swamy Yeleswaram are at Incyte Corporation, Wilmington, Delaware, USA. Correspondence to S.Y.

e-mail:yeleswaram@incyte.com

doi: $10.1038 / \mathrm{nrd} 3028-\mathrm{c} 1$

1. The International Transporter Consortium. Membrane transporters in drug development. Nature Rev. Drug Discov. 9, 215- 236 (2010)

2. Lanoxin (Digoxin) Elixir Pediatric Product Information [online], < http://us.gsk.com/products/assets/us lanoxin elixir.pdf $>$ (2010)

3. Huffman, D. H. \& Azarnoff, D. L. Absorption of orally given digoxin preparations. J. Amer. Med. Assoc. 222, 957-960 (1972)

4. Reisswll, P., Ojala, K., Manninen V. \& Sothman, A. Correlation of digoxin-tablet dissolution-rate with biological availability. Br. J. Clin. Pharmacol. 4 , 235- 236 (1977).

5. Yu, L.. X. An integrated model for determining causes for poor oral drug absorption. Pharm. Res.16, 1883-1887 (1999).

6. Becquemont, L. et al. Effect of grapefruit juice on digoxin pharmacokinetics in humans. Clin. Pharmacol. Ther. 70, 311-316 (2001)

7. Swarbrick, J. Encyclopedia of Pharmaceutical Technology 3rd edn, Vol. 5, 3335 (Informa Healthcare, New York, 2007).
8. Lin, J. H. \& Yamazaki, M. Clinical relevance of P-glycoprotein in drug therapy. Drug Metab. Rev. 35 417-454 (2003)

9. Tayrouz $\mathrm{Y}$ et al. Pharmacokinetic and pharmaceutic interaction between digoxin and cremophor $\mathrm{RH} 40$. Clin. Pharmacol. Ther. 73, 397-405 (2003).

10. Wetterich, U. et al. Evidence for intestinal secretion as an additional clearance pathway of talinolol enantiomers: concentration- and dose-dependent absorption in vitro and in vivo. Pharm. Res. 13, 514-522 (1996)

11. Trausch, B., Oertel, R., Richter, K. \& Gramatté, T. Disposition and bioavailability of the $\beta 1$-adrenoceptor antagonist talinolol in man. Biopharmaceut. Drug Disp. 16, 403-414 (1995).

12. Tubic, M. et al. Effects of controlled-release on the pharmacokinetics and absorption characteristics of a compound undergoing intestinal efflux in humans. Eur. J. Pharm. Sci. 29, 231-239 (2006).

13. Schwarz, U. I., Gramatté, T., Krappweis, J., Oertel, R. \& Kirch, W. P-glycoprotein inhibitor erythromycin increases oral bioavailability of talinolol in humans. Int. J. Clin. Pharmacol. Ther. 38, 161-167 (2000).

14. Westphal, K., Weinbrenner, A., Zschiesche, M., et al. Induction of P-glycoprotein by rifampin increases intestinal secretion of talinolol in human beings: A new type of drug/drug interaction. Int. J. Clin. Pharmacol. Ther. 68, 345-355 (2000)

15. Schwarz, U. I. et al. Grapefruit juice ingestion significantly reduces talinolol bioavailability. Clin. Pharmacol. Ther. 77, 291-301 (2005). 